\title{
Diagnóstico de Glaucoma em Imagens de Fundo de Olho utilizando os Índices de Diversidade de Shannon e McIntosh
}

\author{
José Denes L. Araújo ${ }^{1}$, Anselmo C. de Paiva ${ }^{1}$, João D. S. de Almeida ${ }^{1}$, \\ Otilio Paulo. S. Neto ${ }^{1}$, Jefferson A. de Sousa ${ }^{1}$, Aristófanes C. Silva ${ }^{1}$, Geraldo Braz Júnior ${ }^{1}$ \\ ${ }^{1}$ Núcleo de Computação Aplicada - Universidade Federal do Maranhão (UFMA) \\ \{josedenes17,alves.jefferson27\}@gmail.com, paiva@deinf.ufma.br \\ otilio.paulo@ifpi.edu.br,\{joao.dallyson, geraldo.braz\}@ufma.br \\ ari@dee.ufma.br
}

\begin{abstract}
Glaucoma is an asymptomatic ocular disease in the early stages that if left untreated can lead to blindness. In most cases it causes an increase in pressure inside the eye (intraocular pressure) causing damage to the optic nerve. The use of image processing techniques for analysis of the fundus of the eye helps experts in the diagnosis of glaucoma, thus preventing vision loss. In this work we propose a method for diagnosis of glaucoma in fundus images using the Shannon and McIntosh diversity indexes as descriptors of texture patterns and support vector machine (SVM) for classification. The application of the Shannon and McIntosh indexes as the texture descriptors proved to be effective, reaching a mean accuracy of $88,35 \%$, a mean sensitivity of $84,50 \%$ and a mean specificity of $91,37 \%$.
\end{abstract}

Resumo. O glaucoma é uma doença ocular assintomática nos estágios iniciais que se não tratada pode levar a cegueira. Na maioria dos casos provoca um aumento da pressão dentro do olho (pressão intraocular) causando lesões no nervo óptico. O uso de técnicas de processamento de imagens para a análise de imagens do fundo do olho auxiliam os especialistas no diagnóstico do glaucoma, prevenindo assim a perda de visão. Neste trabalho é proposto um método para diagnóstico do glaucoma em imagens de fundo de olho utilizando os índices de diversidade de Shannon e McIntosh como descritores dos padrões de textura e support vector machine (SVM) para classificação. A aplicação dos indices de Shannon e McIntosh como descritores de textura mostrou-se eficaz alcançando como melhor resultado uma acurácia média de $88,35 \%$, uma sensibilidade média de 84,50\% e uma especificidade média de 91,37\%.

\section{Introdução}

O glaucoma é uma doença ocular que provoca lesão no nervo óptico e estreitamento sucessivo do campo visual dos pacientes afetados. Uma das causas do glaucoma é a obstrução do escoamento de um líquido, chamado humor aquoso, que existe dentro do olho, causando assim aumento da pressão intraocular. É assintomático nas fases iniciais. O paciente percebe os sintomas apenas em fase avançada quando ocorre a perda irreversível do campo visual e, se não for tratada pode, levar a cegueira [CBO 2017].

Atualmente é a segunda maior causa de cegueira no mundo [WGA 2017]. Estimase que 2 a 3\% da população brasileira acima de 40 anos possam ter a doença [CBO 2017]. 
Seu diagnóstico é baseado principalmente no histórico médico do paciente, aferição da pressão intraocular, teste de perda do campo visual e avaliação manual do disco óptico através de oftalmoscopia ou imagens de fundo de olho [Lin et al. 2007].

O glaucoma causa alterações morfológicas típicas da cabeça do nervo óptico (disco óptico) e camada de fibras nervosas que podem ser verificadas para auxiliar no diagnóstico. Essas alterações ocorrem no formato e tamanho do disco óptico e escavação, área e configuração do anel neural, configuração dos vasos sanguíneos no disco óptico e atrofias na região peripapilar [SBG 2009].

O exame de imagem do fundo de olho é um meio não invasivo, de baixo custo e pode ser usado para diagnosticar diferentes anormalidades oculares como o glaucoma. A análise de imagens digitais do fundo do olho, baseada em técnicas computacionais, é importante na avaliação do desenvolvimento natural da doença [Faust et al. 2012].

O objetivo deste trabalho é propor um novo método capaz de auxiliar especialistas na realização do diagnóstico do glaucoma em imagens de fundo de olho. $\mathrm{O}$ método está organizado em três etapas: pré-processamento, extração de características e classificação. O pré-processamento das imagens é realizado utilizando-se o algoritmo retinex [Banić and Lončarić 2013], separação dos canais da imagem, equalização de histograma, quantização e segmentação do disco óptico utilizando uma marcação fornecida pela base de imagens. Para a extração de características realiza-se a divisão espacial do disco óptico e a aplicação dos índices de diversidade de Shannon [Shannon 1949] e McIntosh [McIntosh 1967] a cada sub-região gerada. Por fim, realiza-se a classificação utilizando SVM.

O restante deste artigo está organizado da seguinte forma. Na Seção 2, são apresentados os trabalhos relacionados ao tema da pesquisa. Na Seção 3, são descritos os materiais e os métodos utilizados neste trabalho. Na Seção 4, são apresentados os resultados obtidos, as discussões sobre esses resultados e um estudo comparativo com outros trabalhos equivalentes ao nosso. Finalizando, na Seção 5 são apresentados as conclusões da pesquisa e os trabalhos futuros em andamento ou a realizar.

\section{Trabalhos Relacionados}

Na literatura existem vários trabalhos relacionados ao problema de diagnóstico de glaucoma utilizando características de textura. A seguir serão apresentados alguns desses trabalhos.

Acharya et al. (2014) propuseram uma metodologia onde a transformada de Gabor é aplicada sobre as imagens e características são extraídas dos coeficientes gerados pela transformada. O número de características é reduzido utilizando Principal Component Analysis (PCA). A classificação é realizada utilizando SVM e Naive Bayesian obtendo-se 93,1\% de acurácia, 89,75\% de sensibilidade e 96,2\% de especificidade.

Já Gajbhiye e Kamthane (2015) propuseram uma metodologia para detecção de glaucoma utilizando transformada de wavelet para decomposição da imagem e higher order moments para cálculo das características. São utilizados os classificadores SVM, k-Nearest Neighbors (KNN) e Error Back-Propagation Training Algorithm (EBPTA). A metodologia alcança uma acurácia de 86,57\%.

$\mathrm{Na}$ metodologia proposta por Haleem et al. (2015) é utilizada a transformada de wavelet aplicada de forma regional no disco óptico. As imagens são divididas em quatro 
quandrantes seguindo a regra ISNT (Inferior, Superior, Nasal e Temporal) e para cada região é calculada a energia wavelet através de diferentes sub-bandas usando diferentes famílias wavelet. A metodologia alcança uma taxa de acerto de 92,95\%.

O método proposto por Salam et al. (2016) é baseada em um conjunto híbrido de características. São extraídas dois tipos de características: estruturais (razão entre disco e escavação), onde é necessária a segmentação do disco óptico e da escavação; e não estruturais (textura e intensidade). A classificação é realizada com SVM obtendo-se uma acurácia de $91 \%$, sensibilidade de $100 \%$ e especifidade de $87 \%$.

Sousa et al. (2016) utilizaram LBP (Local Binary Pattern) para representar a região do disco óptico, funções geoestatísticas (semivariograma, semimadograma, covariograma e correlograma) para extrair características de textura e SVM para a classificação alcançando-se $84 \%$ de acurácia, $82 \%$ de sensibilidade e $88 \%$ de especificidade.

Neste trabalho utiliza-se o conceito de índices de diversidade que são aplicados na ecologia para medir a diversidade de espécies em comunidades e realizar a comparação das diversidades entre as comunidades. Os índices de diversidade de Shannon e McIntosh são adaptados para serem utilizados como descritores de textura aplicados em imagens de fundo de olho com a finalidade de diagnóstico de glaucoma.

\section{Materiais e Métodos}

Esta seção apresenta o método proposto para diagnosticar o glaucoma em imagens de fundo de olho. A Figura 1 apresenta as etapas deste método. Para a realização dos testes é utilizada a base de imagens RIM-ONE [Trucco et al. 2013]. A primeira etapa do método é o pré-processamento (Seção 3.2). A segunda etapa é a extração de característica (Seção 3.3) utilizando os índices de diversidade de Shannon e McIntosh (Seção 3.3.1). Por último é realizada a classificação com SVM e a validação dos resultados (Seção 3.4).

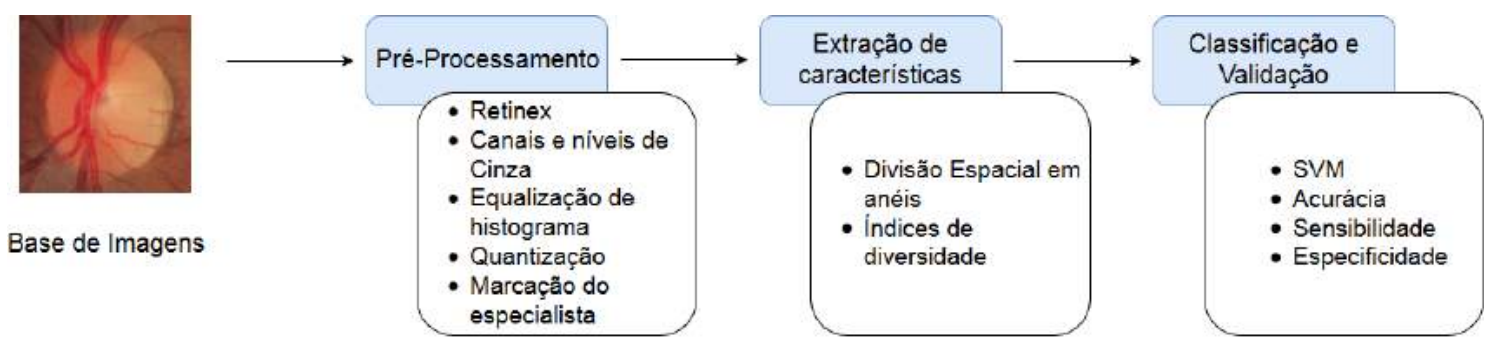

Figura 1. Etapas do método

\subsection{Base de Imagens}

A base de imagens de fundo de olho utilizada neste trabalho é a RIM-ONE $v 2$ que é disponibilizada com imagens fornecidas por três hospitais: Hospital Universitário de Canarias, Hospital Clínico San Carlos e Hospital Universitário Miguel Servet. Possui 455 imagens separadas em duas classes. A classe Normal, possui 255 imagens de retinas saudáveis, e a classe Glaucoma and suspicious possui 200 imagens de retinas com glaucoma ou suspeitas. Algumas das imagens da classe Glaucoma and suspicious, por não apresentarem o diagnóstico claro e definitivo, são semelhantes as imagens da classe Normal.

As imagens são fornecidas no formato JPEG, sistema de cor RGB e acompanhadas por uma marcação, indicando a região do disco óptico, feita por um especialista. A Figura 
2 (a) apresenta exemplo de imagem da classe Normal e a Figura 2 (b) da classe Glaucoma and suspicious acompanhadas de suas respectivas marcações do disco óptico.

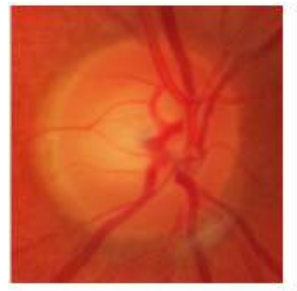

(a) Normal

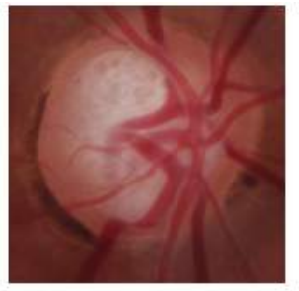

(b) Glaucoma and Suspicious

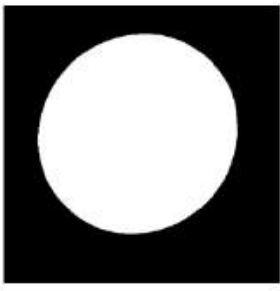

(a) classe Normal e (b) classe

\subsection{Pré-Processamento}

A preparação das imagens para a extração de características é feita em cinco subetapas. Na primeira subetapa utiliza-se o retinex [Banić and Lončarić 2013] para corrigir a iluminação não uniforme das imagens da retina. Na segunda subetapa a imagem que encontra-se no sistema de cor RGB é dividida nos seus respectivos canais (Red, Green e Blue) e também é gerada uma imagem em níveis de cinza (totalizando quatro imagens). Foi testada a aplicação do método a cada uma das imagens separadamente e combinadas. Porém a junção das características das quatro imagens apresentou melhor resultado.

Em seguida, na terceira subetapa, aplica-se a equalização de histograma nas imagens com o objetivo de realçar o contraste das estruturas da retina e fornecer uma melhor descrição da textura. A Figura 3 (a) mostra uma imagem em níveis de cinza antes da equalização e a Figura 3 (b) após a equalização.

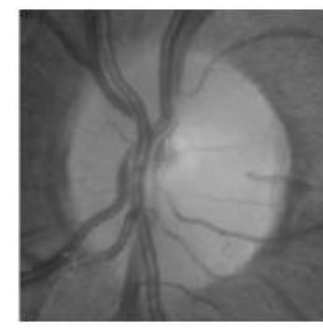

(a) Imagem em niveis de cinza

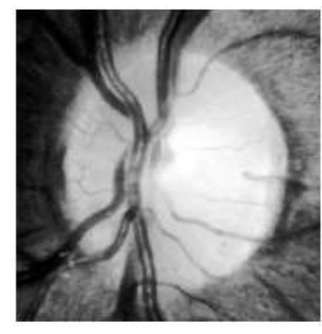

(b) Imagem em níveis de cinza equalizada

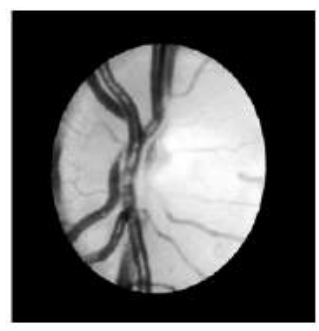

(c) Região do disco óptico

Figura 3. Equalização de histograma aplicada a imagem de fundo de olho e segmentação do disco óptico: (a) imagem em níveis de cinza, (b) imagem em níveis de cinza equalizada e (c) região do disco óptico

$\mathrm{Na}$ quarta subetapa, cada uma das quatro imagens equalizadas é decomposta em outras seis imagens com valores máximos diferentes (gerando quantizações diferentes). São usadas como faixas de valores de quantização: 8, 7, 6, 5, 4, e 3 bits. A quantização é utilizada para combinar os indivíduos (pixels) em um número menor de espécies (intensidades). Assim, aumentando a capacidade de capturar informação sobre indivíduos de baixa ocorrência ao serem unidos com outros indivíduos. Na quinta e última subetapa de pré-processamento a máscara fornecida pela RIM-ONE $v 2$ é aplicada a cada imagem para a obtenção da região do disco óptico (região onde as características serão extraídas). A Figura 3 (c) mostra o resultado da aplicação da máscara na imagem. 


\subsection{Extração de Características}

Após o pré-processamento as imagens são submetidas a extração de características. Os índices de diversidade de Shannon e McIntosh (Seção 3.3.1) são utilizados como descritores de textura. Para aplicação dos índices uma divisão espacial do disco óptico (Seção 3.3.2) é realizada, permitindo uma análise de cada sub-região obtida.

\subsection{1. Índices de Diversidade}

Diversidade, no contexto de ecologia, refere-se a variedade de espécies em uma comunidade ou habitat. Biodiversidade envolve dois conceitos: riqueza de espécies e equabilidade. Riqueza de espécie é simplesmente o número de espécies presentes em uma determinada comunidade ou área de interesse. Equabilidade descreve a variabilidade na abundância de espécies. Em uma comunidade em que todas as espécies tem aproximadamente o mesmo número de indivíduos sua equabilidade é máxima. Um índice de diversidade é uma estatística que incorpora informações de riqueza e equabilidade. Esse é utilizado para medir a diversidade de uma comunidade onde cada membro pertence a uma única espécie. [Marrugan 2004].

Os índices de diversidade são utilizados em situações comparativas. Pode-se utilizá-los para dizer que a comunidade $A$ possui uma maior ou menor diversidade que a comunidade $B$ [Melo 2008]. Para entender as equações que calculam os índices são necessárias algumas definições: $S$ é o número de espécies presentes na amostra, $N$ é o número total de indivíduos da amostra, $n_{i}$ é o número de indivíduos da espécie $i$ e $p_{i}$ é a probabilidade de um indivíduo pertencer a espécie $i$ e é calculado por $p_{i}=n_{i} / N$.

O índice de Shannon é derivado da teoria da informação e assume que indivíduos são amostrados aleatoriamente de uma comunidade infinitamente grande e que todas as espécies estão representadas na amostra [Marrugan 2004]. É definido pela equação:

$$
H=-\sum_{i=1}^{S} p_{i} \ln p_{i}
$$

O índice de McIntosh parte do princípio que uma comunidade pode ser vista como um ponto em um hiper-volume S-dimensional e a distância euclidiana da comunidade para a origem pode ser utilizada como uma medida de diversidade. É uma medida de probabilidade de dois indivíduos selecionados aleatoriamente de uma comunidade infinitamente grande pertencerem a mesma espécie [Marrugan 2004]. É definido pela equação:

$$
M c=\frac{N-U}{N-\sqrt{N}}
$$

onde $U=\sqrt{\sum_{i=1}^{S} n_{i}^{2}}$ é a distância euclidiana da comunidade para a origem.

Este trabalho investiga o uso dos índices de diversidade de Shannon e McIntosh, como descritores de textura, para diferenciar imagens de retinografias como saudáveis ou glaucomatosas. Para realizar essa investigação deve-se considerar os pixels como indivíduos e suas tonalidades representando os conjuntos de espécies. 


\subsubsection{Divisão Espacial da Imagem}

Cada imagem gerada pela etapa de pré-processamento é dividida em sub-regiões em forma de anéis. Para a divisão da região primeiro são calculados círculos concêntricos. Para isso encontra-se o valor do raio do maior círculo que é calculado medindo-se a distância do centro do disco óptico até o pixel da borda mais distante. Para obter os valores dos raios de cada círculo concêntrico é utilizada a equação $R_{i}=i * R_{\max } / \mathrm{nr}$ onde $R_{i}$ é o raio do i-ésimo círculo, $R_{\max }$ é o valor do maior raio, $i$ é o índice do raio do círculo a ser determinado e $n r$ é o total de círculos que cada imagem será dividida.

A partir dos círculos são obtidos as sub-regiões em forma de anéis. Um anel é a área representada pela diferença entre dois círculos consecutivos pois esses compartilham o mesmo centro. A Figura 4 mostra as sub-regiões em forma de anéis geradas a partir do disco óptico. A última imagem da esquerda para a direita é o último anel obtido e possui uma forma diferente pois seu limite externo é a marcação do disco óptico.
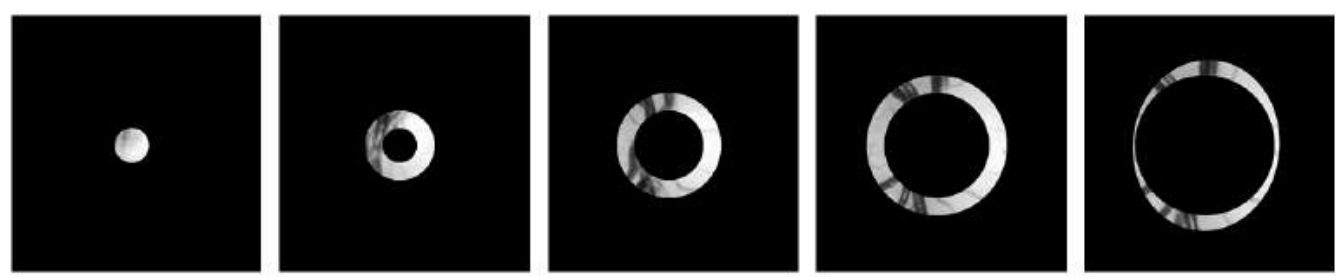

Figura 4. Exemplo de sub-regiões em forma de anéis

\subsection{Classificação e Validação dos Resultados}

A última etapa do método é a classificação das imagens em saudáveis e glaucomatosas. As características geradas na etapa de extração são submetidas a SVM com função de base radial (RBF), kernel mais comumente usado para problemas de reconhecimento de padrões. Todas as características foram normalizadas entre -1 e 1 para melhorar a performance. Assim, garante-se um tempo de processamento mais curto sem descaracterizar o valor original da característica [Duda and Hart 1973].

A SVM [Vapnik 1998] é um método para estimar uma função de classificação dos dados baseada na construção de um hiperplano como superfície de decisão de forma que a margem de separação entre as classes seja maximizada. Utiliza o princípio de minimização do risco estrutural, que se baseia no fato de que a taxa de erro de uma máquina de aprendizado nos dados de teste é limitada pela soma da taxa de erro de treinamento e por um termo que depende da dimensão Vapnik-Chervonenkis (dimensão VC) [Zhuang and Dai 2006].

Na SVM a superfície de decisão é definida pela equação:

$$
f(x)=\sum_{i=1}^{l} \alpha_{i} y_{i} k\left(x, x_{i}\right)+b
$$

onde $k\left(x, x_{i}\right)=\Phi(x) . \Phi\left(x_{i}\right)$ é a função de kernel ( $\Phi$ representa a transformação dos dados de entrada para um espaço de característica de maior dimensão), e os coeficientes $\alpha_{i}$ e $b$, são soluções das equações: 


$$
\min _{w, b, \xi} \frac{1}{2} w^{T} \cdot w+C \sum_{i=1}^{l} \xi_{i}
$$

sujeito a:

$$
y_{i}\left[w^{T} \cdot \phi\left(x_{i}\right)+b\right] \geq 1-\xi_{i}
$$

onde $w$ é um vetor de pontos perpendicular no hiperplano de separação, $C>0$ é um parâmetro escolhido pelo usuário, correspondendo a penalidade do erro e os $\xi_{i}$ 's são variáveis de folga que penalizam os erros de treinamento. Funções de kernel são utilizadas pela SVM para lidar com distribuição de dados que possuem separação de limites complexa. Os dados no espaço de características são transformados para outro espaço de maior dimensão onde é possível criar um hiperplano de separação. A RBF é definida pela equação $k(x, y)=e^{-\gamma\|x-y\|^{2}}$ onde $\gamma>0$ é um parâmetro escolhido pelo usuário.

O resultado da classificação é identificar as imagens como saudáveis ou glaucomatosas. Quando uma imagem com glaucoma é corretamente classificada é chamada de verdadeiro positivo (VP). Uma imagem saudável classificada corretamente é chamada de verdadeiro negativo (VN). Quando uma imagem glaucomatosa é considerada saudável é chamada falso negativo (FN). E quando uma imagem saudável é considerada glaucomatosa é chamada falso positivo (FP) [Chimieski and Fagundes 2013].

A partir desses valores as métricas de sensibilidade, especificidade e acurácia são calculadas para avaliar o desempenho do classificador. A sensibilidade indica a porcentagem de vezes que o teste acertou imagens glaucomatosas e é definida como $V P /(V P+F N)$. A especificidade é a porcentagem de vezes que o teste acertou imagens saudáveis e é definida como $V N /(V N+F P)$. A acurácia é a porcetagem de casos classificados corretamente sobre o número total de casos e é definida como $(V P+V N) /(V P+F P+V N+F N)$.

\section{Resultados e Discussões}

Nesta seção são apresentados os resultados alcançados pelo método proposto na Seção 3. Foram submetidas ao método todas as imagens da base (Seção 3.1). Essas passaram primeiramente pela etapa de pré-processamento (Seção 3.2). Cada imagem préprocessada originou um total de vinte e quatro imagens (4 imagens (R, G, B, Gray scale) * 6 quantizações). Cada imagem gerada é dividida em 5 sub-regiões (valor escolhido empiricamente) em forma de anéis (Seção 3.3.2). As características são extraídas de cada sub-região. Para cada índice de diversidade são extraídas 120 características (4 imagens (R, G, B, Gray scale) * 6 quantizações * 5 anéis).

Para a classificação foi utilizada a SVM com RBF como kernel, onde seus dois parâmetros, $C$ e $\gamma$, foram estimados utilizando-se o grid-search [Chang and Lin 2011]. $\mathrm{Na}$ classificação a base de imagens foi dividida aleatoriamente em treinamento e teste. As porcentagens utilizadas foram 50/50\%, 60/40\%, 70/30\% e 80/20\% para treinamento e teste respectivamente realizando cinco classificações para cada. Essa configuração de número de proporções e repetições tem o objetivo de mostrar que o método é robusto a diversas situações. As métricas de sensibilidade, especificidade e acurácia foram utilizadas para avaliar cada classificação. Os parâmetros $C$ e $\gamma$ foram estimados para cada novo conjunto de treinamento. 
Para os testes os índices de diversidade são utilizados separadamente e em conjunto. A média de cada métrica, após cinco classificações, acompanhada do desvio padrão são apresentadas nas Tabelas 1, 2 e 3. Pode-se observar na Tabela 1 que para o índice de Shannon o melhor resultado foi obtido na proporção 70/30\% com uma acurácia média de $85,69 \%$, uma sensibilidade média de $83,00 \%$ e especificidade média de $87,79 \%$.

Tabela 1. Resultados dos testes utilizando o índice de Shannon

\begin{tabular}{c|c|c|c}
\hline Treinamento/Teste (\%) & Acurácia (\%) & Sensibilidade (\%) & Especificidade (\%) \\
\hline $50 / 50$ & $80,00 \pm 0,03$ & $79,60 \pm 0,03$ & $80,31 \pm 0,04$ \\
\hline $60 / 40$ & $81,87 \pm 0,03$ & $82,75 \pm 0,06$ & $81,18 \pm 0,07$ \\
\hline $\mathbf{7 0 / 3 0}$ & $\mathbf{8 5 , 6 9} \pm 0,04$ & $\mathbf{8 3 , 0 0} \pm 0,08$ & $\mathbf{8 7 , 7 9} \pm 0,03$ \\
\hline $80 / 20$ & $84,40 \pm 0,05$ & $81,00 \pm 0,10$ & $87,06 \pm 0,03$ \\
\hline
\end{tabular}

Para os testes com o índice de McIntosh (Tabela 2) o melhor resultado foi obtido na proporção 80/20\% com uma acurácia média de 83,52\%, uma sensibilidade média de $81,00 \%$ e especificidade média de $85,49 \%$. Nos testes realizados utilizando a junção dos índices de Shannon e McIntosh, o melhor resultado foi obtido na proporção 80/20 como pode ser visto na Tabela 3 , com uma acurácia média de $88,35 \%$, sensibilidade média de $84,50 \%$ e especificidade média de $91,37 \%$.

Tabela 2. Resultados dos testes utilizando o índice de Mclntosh

\begin{tabular}{c|c|c|c}
\hline Treinamento/Teste $(\%)$ & Acurácia (\%) & Sensibilidade (\%) & Especificidade (\%) \\
\hline $50 / 50$ & $78,77 \pm 0,02$ & $82,80 \pm 0,04$ & $75,63 \pm 0,04$ \\
\hline $60 / 40$ & $79,89 \pm 0,04$ & $82,50 \pm 0,02$ & $77,84 \pm 0,07$ \\
\hline $70 / 30$ & $83,36 \pm 0,01$ & $80,67 \pm 0,05$ & $85,45 \pm 0,03$ \\
\hline $\mathbf{8 0 / 2 0}$ & $\mathbf{8 3 , 5 2} \pm 0,03$ & $\mathbf{8 1 , 0 0} \pm 0,07$ & $\mathbf{8 5 , 4 9} \pm 0,03$ \\
\hline
\end{tabular}

Tabela 3. Resultados dos testes utilizando os índices de Shannon e Mclntosh

\begin{tabular}{c|c|c|c}
\hline Treinamento/Teste (\%) & Acurácia (\%) & Sensibilidade (\%) & Especificidade (\%) \\
\hline $50 / 50$ & $81,32 \pm 0,03$ & $83,20 \pm 0,06$ & $79,84 \pm 0,04$ \\
\hline $60 / 40$ & $82,97 \pm 0,04$ & $84,00 \pm 0,06$ & $82,16 \pm 0,08$ \\
\hline $70 / 30$ & $82,92 \pm 0,06$ & $81,67 \pm 0,08$ & $83,90 \pm 0,05$ \\
\hline $\mathbf{8 0 / 2 0}$ & $\mathbf{8 8 , 3 5} \pm 0,04$ & $\mathbf{8 4 , 5 0} \pm 0,05$ & $\mathbf{9 1 , 3 7} \pm 0,04$ \\
\hline
\end{tabular}

O método apresentou valores de especificidades no geral maiores que os de sensibilidade indicando um acerto maior de casos de imagens saudáveis. Isso pode ser explicado pelo fato da classe Glaucoma and suspicious possuir imagens suspeitas de glaucoma que são semelhantes as imagens da classe Normal. Os resultados apresentados demonstram que as características geradas pela aplicação do índice de Shannon combinadas com o índice de McIntosh conseguem classificar de forma eficaz as imagens de fundo de olho. Os desvios padrões das métricas, em todos os testes, apresentam valores menores que um, indicando que o método possui pouca variação entre as execuções.

A Tabela 4 mostra uma breve comparação dos resultados encontrados neste trabalho com alguns trabalhos relacionados. São apresentadas a base utilizada, quantidade de amostras, e as métricas de validação. Este trabalho comparado aos que usam a mesma base de imagens possui resultado melhor que os trabalhos de Gajbhiye e Kamthane (2015) 
e Sousa et al. (2016) que utilizam um número de imagens menor e igual respectivamente em relação ao método proposto. Possui resultados inferiores ao trabalho de Haleem et al. (2015) que utiliza um número menor de imagens. Comparado com trabalhos que utilizam outras bases possui resultados inferiores ao trabalho de Salam et al. (2016), porém esse utiliza um número menor de imagens. A maioria dos trabalhos relacionados utilizou uma base com um número menor de amostras, exceto o trabalho de Acharya et al. (2014) que também possui os melhores resultados entre todos os trabalhos.

Tabela 4. Comparação do método com os trabalhos relacionados. Acurácia (Ac), Sensibilidade (Sen) e Especificidade (Esp)

\begin{tabular}{c|c|c|c|c}
\hline Trabalho & Base (amostras) & Ac (\%) & Sen (\%) & Esp (\%) \\
\hline Acharya et al., 2014 & privada (510) & 93,1 & 89,75 & 96,2 \\
\hline Gajbhiye and Kamthane, 2015 & RIM-ONE (350) & 86,57 & - & - \\
\hline Haleem et al., 2015 & RIM-ONE (158) & 92,95 & - & - \\
\hline Salam et al., 2016 & privada (150) & 91 & 100 & 87 \\
\hline Sousa et al. 2016 & RIM-ONE (455) & 84 & 82 & 88 \\
\hline Método Proposto & RIM-ONE (455) & $\mathbf{8 8 , 3 5}$ & $\mathbf{8 4 , 5 0}$ & $\mathbf{9 1 , 3 7}$ \\
\hline
\end{tabular}

\section{Conclusão e Trabalhos Futuros}

Neste trabalho foi proposto um método para o diagnóstico de glaucoma utilizando os índices de diversidade de Shannon e McIntosh para a extração de características da textura e SVM para classificação das imagens em glaucomatosas ou saudáveis. O glaucoma é a segunda maior causa de cegueira no mundo. Seu diagnóstico em estágios iniciais previne a perda de visão e cegueira através da disponibilização de métodos mais eficientes de tratamento.

Os resultados obtidos pelo método proposto são compatíveis aos apresentados na revisão de literatura e comprovam que os índices de diversidade de Shannon e McIntosh podem ser utilizados para caracterizar textura de imagens de fundo de olho. Como trabalhos futuros, pretende-se: implementar e testar outros índices de diversidade (Brillouin, Berger Parker, Simpson e Camargo) para melhorar as taxas de acerto; utilizar outras técnicas de reconhecimento de padrões (Random Forest, Adaboost e Naive Bayesian); e aplicar esse método a outras bases de imagens de fundo de olho.

\section{Referências}

Acharya, U. R., Ng, E. Y. K., Eugene, L. W. J., Noronha, K. P., Min, L. C., Nayak, K. P., and Bhandary, S. V. (2014). Decision support system for the glaucoma using Gabor transformation. Biomedical Signal Processing and Control, 15:18-26.

Banić, N. and Lončarić, S. (2013). Light random sprays retinex: Exploiting the noisy illumination estimation. IEEE Signal Processing Letters, 20(12):1240-1243.

CBO (2017). Glaucoma. http: //www. cbo.net.br/novo/publico-geral/ glaucoma. php. (Acesso em 26 fev. 2017).

Chang, C.-C. and Lin, C.-J. (2011). LIBSVM: A library for support vector machines. ACM Transactions on Intelligent Systems and Technology, 2:27:1-27:27. 
Chimieski, B. F. and Fagundes, R. D. R. (2013). Association and classification data mining algorithms comparison over medical datasets. Journal of health informatics, $5(2)$.

Duda and Hart (1973). Pattern classification and scene analysis. John Wiley.

Faust, O., Acharya, R., Ng, E. Y.-K., Ng, K.-H., and Suri, J. S. (2012). Algorithms for the automated detection of diabetic retinopathy using digital fundus images: a review. Journal of medical systems, 36(1):145-157.

Gajbhiye, G. O. and Kamthane, A. N. (2015). Automatic classification of glaucomatous images using wavelet and moment feature. In 2015 Annual IEEE India Conference (INDICON), pages $1-5$.

Haleem, M. S., Han, L., van Hemert, J., and Fleming, A. (2015). Glaucoma classification using regional wavelet features of the onh and its surroundings. In Engineering in Medicine and Biology Society (EMBC), 2015 37th Annual International Conference of the IEEE, pages 4318-4321. IEEE.

Lin, S. C., Singh, K., Jampel, H. D., Hodapp, E. A., Smith, S. D., Francis, B. A., Dueker, D. K., Fechtner, R. D., Samples, J. S., Schuman, J. S., et al. (2007). Optic nerve head and retinal nerve fiber layer analysis: a report by the american academy of ophthalmology. Ophthalmology, 114(10):1937-1949.

Marrugan, A. (2004). Measuring biological diversity. Victoria, Australia: Blackwell Scienc Ltd a Blackwell Publishing company.

McIntosh, R. P. (1967). An index of diversity and the relation of certain concepts to diversity. Ecology, 48(3):392-404.

Melo, A. S. (2008). O que ganhamos confundindo riqueza de espécies e equabilidade em um índice de diversidade. Biota Neotropica, 8(3):21-27.

Salam, A. A., Khalil, T., Akram, M. U., Jameel, A., and Basit, I. (2016). Automated detection of glaucoma using structural and non structural features. SpringerPlus, 5(1):1519.

SBG (2009). $3^{\mathrm{a}}$ consenso brasilleiro de glaucoma de ângulo aberto. http: / / www . sbglaucoma.com.br/pdf/consenso03.pdf. (Acesso em 01 mar. 2017).

Shannon, C. E. (1949). W. weaver the mathematical theory of communication. Urbana: University of Illinois Press, 29.

Sousa, J. A. d., Almeida, J. D. S., Paiva, A. C. d., Silva, A. C., and Gattass, M. (2016). Diagnóstico de glaucoma em retinografias baseado em geoestatística. Journal of Health Informatics, 8:737-746.

Trucco, E., Ruggeri, A., Karnowski, T., Giancardo, L., Chaum, E., Hubschman, J. P., Al-Diri, B., Cheung, C. Y., Wong, D., Abramoff, M., et al. (2013). Validating retinal fundus image analysis algorithms: Issues and a proposalvalidating retinal fundus image analysis algorithms. Investigative ophthalmology \& visual science, 54(5):3546-3559.

Vapnik, V. (1998). Statistical Learning Theory. Wiley New York.

WGA (2017). What is glaucoma? http://www.worldglaucoma.org/ what-is-glaucoma/. (Acesso em 17 mar. 2017).

Zhuang, L. and Dai, H. (2006). Parameter optimization of kernel-based one-class classifier on imbalance learning. Journal of Computers, 1(7):32-40. 